\title{
EVALUATION OF DIFFRACTION DATA FROM ELECTRON DIFFRACTION PATTERNS OF NATURAL POLYMER MICROCRYSTALS
}

\author{
D. MILLER* , H. CHANZY* ${ }^{*}$ and G. PARAdos $\mathrm{I}^{* * *}$ \\ "Department of Physics, Clemson University, Clemson, SC 29634, \\ U.S.A. \\ * "Centre de Recherche sur Macromolécules Végétales, \\ F-38042 Grenoble, France \\ "* Universita di Napoli, I-80138 Napoli, Italia
}

\begin{abstract}
Résumé - Une méthode de traitement de données de diffraction électronique sur film est présentêe. La réduction du bruit est obtenue par calcul de la moyenne des valeurs des données correspondant à des points équivalents par symétrie sur la figure de diffraction et par calcul d'une moyenne locale sur 3 points voisins. La soustraction globale du bruit de fond est effectuée après évaluation de celui-ci sous forme de développement polynomial dans le cas d'une approximation de moyenne radiale, sinon sous forme de développement en fonctions splines à 2 dimensions; cette évaluation utilise les données situées entre les pics. L'intégration des pics est faite par une méthode d'autoconvolution appliquée soit aux valeurs d'intensité de chaque pic pris isolément soit à 1 'ensemble des données. Le programme a été conçu pour être d'utilisation facile et fonctionne à 1 'aide d'un menu. Il devrait être utilisable avec un microscope électronique équipé d'un ordinateur et d'un détecteur à localisation spatiale à 2 dimensions.
\end{abstract}

Abstract - Methods of evaluating electron diffraction data from films is discussed. Noise reduction is accomplished by heuristic averaging of symmetry related data and 3-point averaging. Global background is removed by radial polynomial fit to intermaxima data or by bivariate splinning. Integration is performed by self-convolution of data subarrays or main array quadrant. A user friendly, menu driven program set is described. The program should be usable in a computer controlled electron microscope with 2-D position sensitive detector.

\section{INTRODUCTION}

The recording of a transmission electron diffraction pattern is, essentially, done in 2-dimensions. For transmission through most samples the accelerating voltage must be large, $100 \mathrm{kV}$ or more. The DeBroglie wavelength of the electrons are thus quite small. The radius of the diffraction (Ewald) sphere, $1 / 2$, is therefore quite large. It is thus possible to record many diffraction maxima simultaneously, a slice through the origin of the reciprocal lattice of a crystalline specimen. Because the specimen is quite small and thin, and the diffraction angles are quite small, the closeness of adjacent diffraction beams often prevents the use of a beamstop. The diffraction pattern is, therefore, registered around a strong incident beam.

The small dimensions of natural polymer microcrystals introduce other complications. Diffraction maxima in the diffraction pattern are only 
several film silver grains in width. Diffraction patterns are thus very granular. The microcrystals are poor conductors electrically and thermally, so beam damage is rapid. Exposures must be made quickly, resulting in considerable noise, both in the background and in the diffraction maxima. It is not uncommon for considerable variation to occur among symmetry related maxima.

A need thus exists for systematic elimination of variations amoung symmetry related maxima (concurrent with eliminatin of unwanted artifacts, such as dust spots, scratches, etc, if possible), removal of the average optical density of beam spallation and background scattering and noise reduction (at least in regions outside maxima). Maxima may require additional local background removal (an example would be radial distribution diffraction rings in the pattern from amorphous phase in the specimen). A rapid integration technique would allow selection of diffraction patterns to be made, based both upon computer intensities and the visual pattern, rather than only upon the latter. Finally, it would be desirable for the data evaluation programs to be written in as portable form as possible and in a language usable even in a microcomputercontrolled electron microscope.

\section{EVALUATION PROGRAM DEVELOPMENT}

In the present instance, electron diffraction patterns were recorded on film. The film was then reduced to a 2-dimensional data array by scanning on a rotary drum photometer.
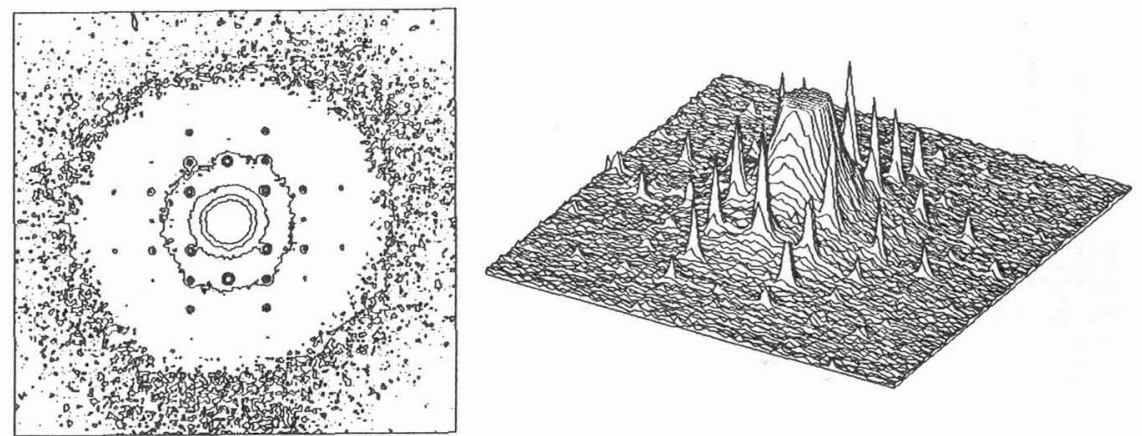

Figure 1. A pseudo 3-D drawing and a contour level drawing of a Mannan 1 diffraction pattern, $(h, k, 0)$ plane. Array size is $(400,400)$. Contour levels are at 40,80,120,160, 200 and 240,255 representing 2.0 O.D.

Considerable noise can be seen in both representations, but particularly in the contour drawing at the 40 level. The beam spallation is not centered on the diffraction spot array. This is caused by the incident beam being not perpendicular to the specimen plane and the specimen being not centered in the beam. Additionally, the specimen may bend, slightly from beam heating. All these effects cause some nonuniformity amoung symmetry related diffraction maxima. The effects can be considerably alleviated by 4-quadrant heuristic averaging point by point in the data array. Each 4 symmetry related data points are averaged. The standard deviation is determined. If one of the four is more than 3 standard deviations from the mean, it is rejected and the remaining three averaged. The average replaces all 4 data. 

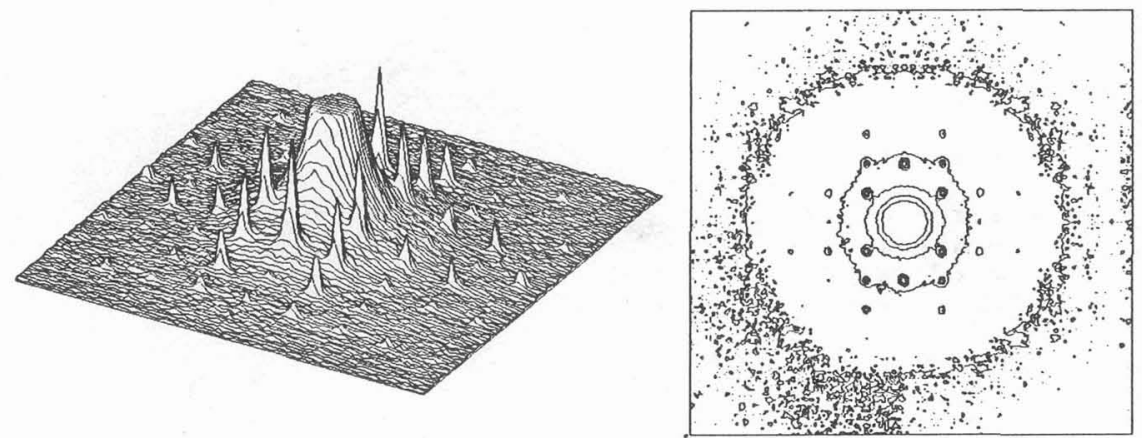

Figure 2. The 4-quadrant average of Figure 1, contour and pseudo 3-D. The lower-left quadrant of the contour drawing has been left undisturbed for comparison.

The reduction in noise is considerable. Notice the several artifacts appearing in only one quadrant of Figure 1. which no longer appear in Figure 2. They have been rejected by the heuristic requirement.

If the pattern is not too acentric or asymmetric and particularly if, in addition, 4-quadrant averaging can be done, beam spallation and global background can be removed by fitting a radial polynomial to background data taken from regions midway between diffraction maxima.

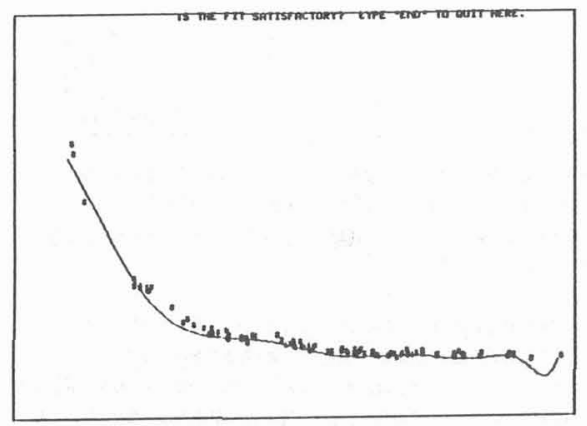

Figure 3. An 8th order Tchebichev polynomial fit to background data.

The fit is good, except at large radius. This, however is at the edge of the diffraction pattern, where few maxima are measurable. If the fit is judged acceptable, polynomial values are computed and subtracted point by point throughout the data array.

Noise between diffraction maxima can be further reduced by heuristic 3-point averaging. If the two neighboring data on opposite sides of a particular datum are of opposite sign, the average of the two replaces the datum. This can be done by moving a 3-datum wide window across the data array, first by rows and then by columns. 


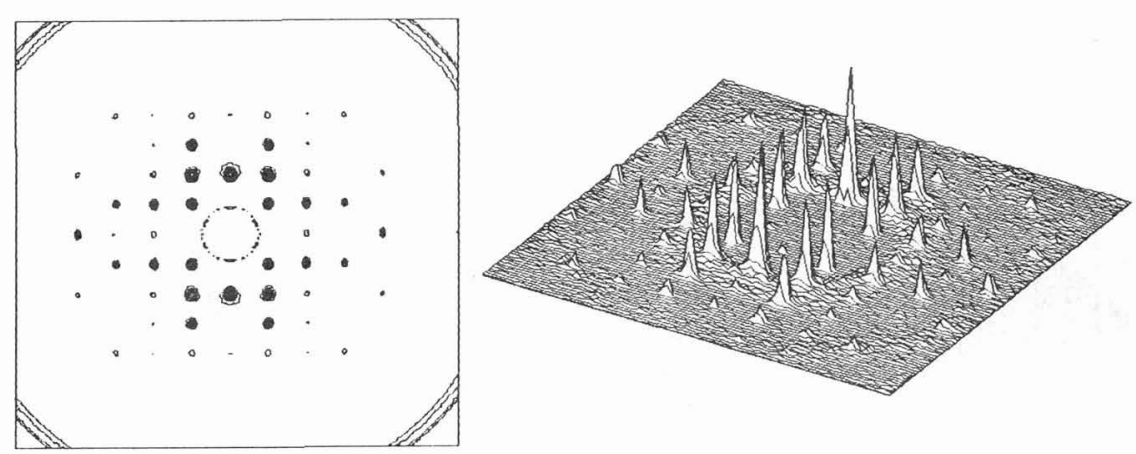

Figure 4. A pseudo 3-D and contour drawing of the Mannan1 pattern after global background removal. Contours every 10 .

Figure 5. The Mannan1 pattern after such noise reduction.

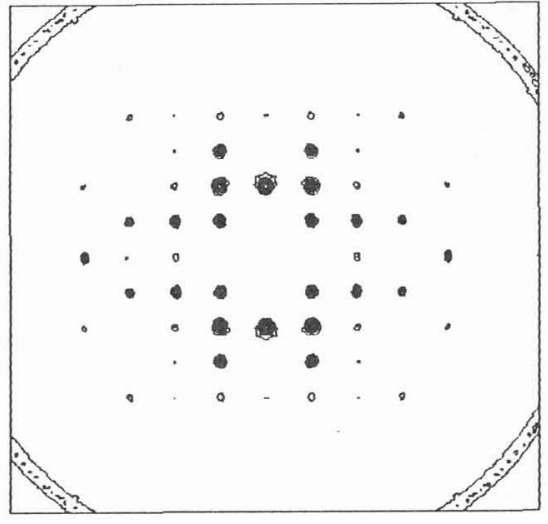

Note that this type of averaging has no effect upon real diffraction maxima, leaving noise in them, nor does it reduce noise in other artifacts, such as the weak, broadened diffraction rings shown in the figure.

In the instance of considerable acentricity of beam and diffraction array, the beam spallation and background can be approximated by a bivariate cubic spline, calculated from data midway between the diffraction maxima. Bivariate splinning, however, requires much more computer CPU time than radial polynomial fits. Splines must be calculated and evaluated for all 160,00 positions of the data array. Such a program, although written and available for interactive use, is presently run offline, at night.

After global background removal has been done, some diffraction maxima may still have local noise, artifacts or background which requires removal. If the object is an artifact or localized noise peak, removal can be accomplished by setting all data within the lowest closed contour of the peak to the value of that contour. If local background requires removal, a local subarray can be removed from the main array. The lowest closed contour of this array can be determined. The data within the subarray exterior to this contour can be used to bivariate spline values within the contour. These values, data and splinned, can be subtracted from the subarray and returned to the main array. 
Integration can be accomplished in a simple fashion, provided that the diffraction maxima covers a sufficient number of scanner steps. The technique is similar to that used in "integrating" $x$-ray diffraction cameras. The local subarray of a diffraction maxima can be removed from the main data array and expanded with zeros to size sufficient to allow step-wise self-convolution to be performed. If this is done, the average value of the maximum region of the self-convolution is proportional to the integration of the diffraction maximum. The standard deviation of the maximum region measures the error of the diffraction maximum. Table 1. shows the integrated intensities of the Mannanl diffraction pattern.

\section{PROGRAMS}

The operations described above have been written as an interactive program system. The following is a portion of the user guide which describes briefly the callable operations.

EDFILMS is a user-friendly, interactive computational system for converting 2-dimensional optical scanner data (in the present circumstance, data from an optronics scanner) from electron diffraction films into diffraction intensities. The programming is primarily in FORTRAN (ansi77). This language was chosen because it is the most widely adopted scientific programming language. Both the main program section and the several subroutines are extensively commented to allow easy additions and reprogramming. Subroutines for graphics, curve and surface fitting and plotting are from two widely available packages: NAG and PLOT1O.

The MAIN program section is written as a monitor which offers the user a choice of the principal task-oriented subroutines (4-letter commandnames) and calls that subroutine. If the user does not know enough to make a choice, he may type LIST. MAIN then presents him with a menu listing and categorizing the subroutines and again offers a choice. Responses should be in Caps. The menu is:

BKGR - Fits a radial polynomial to background data (from between diffraction spots), calculates background and removes it from 'arrayl'.

BKGS - Bivariate splines the background (data from between

diffraction spots) and removes it from 'array1'.

CONT - Displays a contoured graphic of the object 'arrayl' or array $2^{\prime}$. If 'arrayl' is $(400,400)$, cursor location of up to 10 artifacts in the contoured array is offered. If is called from subroutine SPOT, the lowest closed contour in 'array2' can be determined.

CNTR - Finds the symmetry center of 'array1', if it is not too asymmetric or off-center.

DAIN - Reads Optronics data from a dataset, attached to file99, and stores it in binary format into another dataset, 'array'. GETA - Reads the data stored in 'array' into 'arrayl'. INTG - Removes remaining background from 'array2' and integrates it.

LIFT - Lifts a subarray out of 'array1' and puts it into 'array2'. LSCN - Graphs the interpolated values of 'arrayl' along a line between endpoints specified by the user. MOVE - Allows the user to permute 'array1' for centering, etc. MULT - Allows the user to store the average of several data arrays in 'arrayb'. A copy is left in 'arrayl'. 
NOIZ - Uses heuristic 3 point averaging to remove noise fiuctuations from the near-zero background of 'arrayl'. It is used after BKGR or BKGS.

PCTR - Writes 'arrayl' to dataset PICTURE in correct format for use by the Optronics Printer.

PS3D - Displays a pseudo 3-D graphics of the object 'arrayl'. QDAV - Replaces 'array1' with an average of its four quadrants. QUIT - Halts the system.

SHow - Lists 'array1' or 'array2' row by row. SPOT - Lifts a subarray out of 'array1'. The user may: 1) Remove artifacts or 2) Remove the local background around a diffraction spot.

STRA - Writes 'arraylor2' into 'ARRAY' or 'SAVARAY', binary format. If the user supplies any other 4-letter word save these, the menu is presented automatically and he is offered a choice to continue or quit.

The program is available in Fortran'77, written for either the DEC VAX/MNS operating system, or for the Honeywell-Bull Multics operating system. Two widely available subroutine packages are used for polynomial fitting, surface splinning and for plotting, NAG, and PLOT10-TCS. Tape copies of the User Guide and Source can be obtained upon request.

\section{ACKNOWLEDGEMENTS :}

Pseudo 3-D utility, PERSPC, was written by Mr.w.J.Coulthard, University of British Columbia, Canada. Mr.C.Upstill, University of Bristol, U.K. wrote the contour subroutine CNTRS. Programming assistance was gratiously given by Msrs.E.Sibut and J.M.Guillou, Centre Interuniversitaire de Calcul de Grenoble, France, by Mdme.M.Dheu of CERMaV, Grenoble and by Mr.D.Latimer, Computer Center, Clemson University, Clemson, SC, USA. This work was partially funded by a grant from the European Molecular Biology Organization. Principal funding was by CERMAV-CNRS and the state of South Carolina. 
Absentee user Ut112 MACRO logged out 05/03/84 1215.1 hfe Thu CPU usage 27 min $26 \mathrm{sec}$, memory usage 448.0 units

Mannani film 18070, plan de base, 3 scans, G. Paradossi

176,176

$$
137,0,4,0,113,4,4
$$

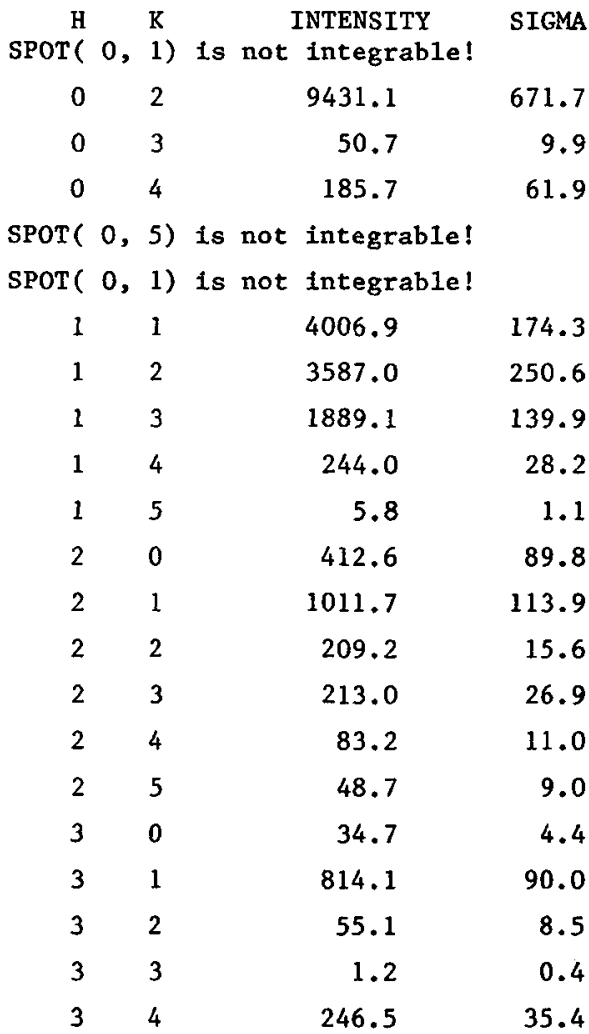

$\operatorname{SPOT}(3,5$ is not integrable!

$\begin{array}{llll}4 & 0 & 1101.6 & 132.1\end{array}$

$\operatorname{SPOT}(4,1)$ is not integrable!

$\begin{array}{llll}4 & 2 & 95.0 & 8.1 \\ 4 & 3 & 31.6 & 7.5\end{array}$

$\operatorname{SPOT}(4,4)$ is not integrable! $\operatorname{SPOT}(4,5)$ is not integrable! 